\title{
Adrenal gland hemorrhage in patients with fatal bacterial infections
}

\author{
Jeannette Guarner ${ }^{1}$, Christopher D Paddock ${ }^{2}$, Jeanine Bartlett ${ }^{2}$ and Sherif R Zaki ${ }^{2}$ \\ ${ }^{1}$ Department of Pathology and Laboratory Medicine, Emory University School of Medicine, Atlanta, GA, USA \\ and ${ }^{2}$ Infectious Diseases Pathology Branch, Division of Viral and Rickettsial Diseases, Center for Disease \\ Control and Prevention, Atlanta, GA, USA
}

\begin{abstract}
A wide spectrum of adrenal gland pathology is seen during bacterial infections. Hemorrhage is particularly associated with meningococcemia, while abscesses have been described with several neonatal infections. We studied adrenal gland histopathology of 65 patients with bacterial infections documented in a variety of tissues by using immunohistochemistry. The infections diagnosed included Neisseria meningitidies, group A streptococcus, Rickettsia rickettsii, Streptococcus pneumoniae, Staphylococcus aureus, Ehrlichia sp., Bacillus anthracis, Leptospira sp., Clostridium sp., Klebsiella sp., Legionella sp., Yersinia pestis, and Treponema pallidum. Bacteria were detected in the adrenal of $40(61 \%)$ cases. Adrenal hemorrhage was present in 39 (60\%) cases. Bacteria or bacterial antigens were observed in $31(79 \%)$ of the cases with adrenal hemorrhage including 14 with $N$. meningitidis, four with $R$. rickettsii, four with $S$. pneumoniae, three with group A streptococcus, two with $S$. aureus, two with $B$. anthracis, one with $T$. pallidum, and one with Legionella sp. Bacterial antigens were observed in nine of $\mathbf{2 6}$ non-hemorrhagic adrenal glands that showed inflammatory foci (four cases), edema (two cases), congestion (two cases), or necrosis (one case). Hemorrhage is the most frequent adrenal gland pathology observed in fatal bacterial infections. Bacteria and bacterial antigens are frequently seen in adrenal glands with hemorrhage and may play a pathogenic role. Although $\mathbf{N}$. meningitidis is the most frequent bacteria associated with adrenal gland pathology, a broad collection of bacteria can also cause adrenal lesions. Modern Pathology (2008) 21, 1113-1120; doi:10.1038/modpathol.2008.98; published online 23 May 2008
\end{abstract}

Keywords: adrenal; hemorrhage; bacteria; infections; immunohistochemistry; N. meningitides

A wide spectrum of adrenal gland pathology is seen during bacterial infections including massive hemorrhage, abscesses, and granulomas. Adrenal hemorrhage is an uncommon condition associated to a variety of infectious and non-infectious diseases. The non-infectious conditions that can cause adrenal hemorrhage include postoperative state, thromboembolic diseases, anticoagulant treatment, burns, trauma, tumor metastasis, and cardiovascular catastrophes. ${ }^{1} \mathrm{~N}$. meningitidis is the most frequent infection associated with adrenal hemorrhage; however, Haemophilus influenzae, Streptococcus pneumoniae, Staphylococcus aureus, group A streptococcus, Neisseria gonorrheae, Pseudomonas aeruginosa, and Klebsiella oxytoca are among other bacteria associated with this pathology. ${ }^{2-10}$ Endotoxinmediated septic shock is the most frequent

Correspondence: Dr J Guarner, MD, Department of Pathology and Laboratory Medicine, Emory University School of Medicine, Egleston Hospital, 1405 Clifton Road, Atlanta, GA 30322, USA. e-mail: Jeanette.Guarner@choa.org

Received 23 January 2008; revised 10 March 2008; accepted 17 March 2008; published online 23 May 2008 clinical condition seen in patients with infectious adrenal hemorrhage. These endotoxins, lypopolysaccharide in the case of Gram-negative bacteria and peptidoglycan for Gram-positive bacteria, bind through toll-like receptors to endothelial and inflammatory cells and induce a number of signaling pathways that lead to transcription of coagulation, fibrinolysis, and proinflammatory cytokine genes such as tissue factor, IL- 1 , IL-6 and $\mathrm{TNF} \alpha^{11}$ In experimental animal models, hemorrhagic adrenal necrosis can be caused by intravenous injection of bacterial endotoxin after pretreatment with adrenocorticotropic hormone. ${ }^{12}$ Although correlation between adrenal hemorrhage and presence of bacteria in the gland has not been described, one report detected $N$. meningitides and $H$. influenzae in six of $11(55 \%)$ adrenal glands of patients with septicemia by using immunofluorescent techniques. ${ }^{13}$

We studied formalin-fixed, paraffin-embedded adrenal glands from cases that were found to have immunohistochemical evidence of a bacterial infection in a variety of tissues in an effort to define the frequency of presence of microorganisms in adrenal glands of patients with fatal bacterial infections and 
the possible role played by bacteria and bacterial antigens in the pathogenesis of adrenal pathology.

\section{Materials and methods}

During 1998-2006, the Centers for Disease Control and Prevention (Atlanta, GA) received 65 consultation cases that were found to have immunohistochemical evidence of a bacterial infection in different tissues and adrenal glands were included in the tissue submitted for diagnostic evaluation. Testing performed in these cases was carried out as a component of public health response. Tissues sent for study and demographic and clinical data varied in completeness from case to case.

Using hematoxylin and eosin stains, adrenal glands were evaluated for the presence of hemorrhage (defined as erythrocytes outside sinusoids), inflammation, edema, and cell necrosis. The amount of hemorrhage and inflammation was assessed as focal when it did not extend beyond a $\times 20$ field and extensive if it was greater than a $\times 20$ field. Kidneys were evaluated for the presence of thrombi and hemorrhage and lungs were evaluated for the presence of hemorrhage and pneumonia. Presence of bacteria was determined by using Brown and Brenn Gram and Steiner silver impregnation stains, and immunohistochemical assays. Immunohistochemical assays for infectious agents have been previously validated and described (Table 1). ${ }^{14-24}$ In summary, assays were performed on deparaffinized, rehydrated $3-\mu \mathrm{m}$ tissue sections. Proteinase $\mathrm{K}$ (Roche, Indianapolis, IN, USA) digestion was carried out for slides treated with all antibodies except for those treated with the anti-Clostridium spp. antibody. Incubation with a primary antibody (Table 1) and colorimetric detection with the LSAB2 Universal alkaline phosphatase kit (Dako, Carpinteria, CA, USA) were performed in the autostainer (Dako). Sections were counterstained with Mayer's hematoxylin (Fisher Scientific, Pittsburgh, PA, USA). Interpretation of immunohistochemical results included the location of the positive reaction and the staining pattern (cocci, bacilli, and granular antigen staining). Systemic disease was defined when immunohistochemical staining was present outside the primary focus of infection. If immunohistochemical staining was only present in the focus of infection it was considered localized, for example, pneumonia.

All primary antibodies were prepared at the Centers for Disease Control and Prevention, except for the antibodies against $S$. aureus (Biodesign, Saco, ME, USA), N. meningitidis (Center for Biologics Evaluation and Research, Food and Drug Administration), Clostridium spp (Biodesign) and K. pneumoniae (Biogenesis, Kingston, NH, USA). Positive controls for the primary antibodies included formalin-fixed, paraffin-embedded bacteria and cases with known bacterial and viral infections
(Table 1). Negative controls consisted of sequential case-patient tissue sections incubated with normal rabbit, dog, or horse serum as the primary antibody.

\section{Results}

Table 2 presents the frequency of infectious agents diagnosed in these 65 patients, their demographic data, the frequency of systemic disease for each microorganism or the syndrome when localized disease was present, and pathological data relating to hemorrhage or thrombosis. None of the cases had an adrenal adenoma. Systemic disease was present in $54(83 \%)$ of 65 cases. Five $(28 \%)$ of the 18 patients with meningococcemia had meningitis. Seven (78\%) of the nine patients with group A streptococcus septicemia had a primary focus of infection including five with a respiratory focus (tonsillitis, laryngeal abscess, or pneumonia), one with necrotizing endometritis, and one with necrotizing fasciitis. The syndromes of the 11 patients with localized disease included pneumonia caused by $S$. pneumoniae (four cases), S. aureus (2), or $K$. pneumoniae (1), necrotizing endometritis caused by Clostridium sordellii (2), mediastinal hemorrhage caused by $B$. anthracis (1), and necrotizing fasciitis due to group A streptococci (1).

Pathologic evidence of adrenal hemorrhage was found in $39(60 \%)$ of 65 cases, all having systemic disease except for two cases (one with pneumonia the other with necrotizing fasciitis). Glomerular thrombi were observed in $13(29 \%)$ of 45 kidneys studied and lung hemorrhages in $10(23 \%)$ of 44 lung specimens available. The frequency of glomerular thrombi and lung hemorrhage by organism is presented in Table 2; of note glomerular thrombi were observed in $50 \%$ of the cases with $N$. meningitidis and group A streptococci while $66 \%$ of the cases with B. anthracis had lung hemorrhage. Fourteen (36\%) of 39 cases with adrenal hemorrhage had glomerular thrombi and hemorrhages in other organs, including seven $(18 \%)$ with glomerular thrombi, four $(10 \%)$ with lung hemorrhages, two (5\%) with kidney hemorrhages, and one with both glomerular thrombi and lung hemorrhages.

Steiner silver impregnation stains showed bacteria (Figure 1a) in nine (15\%) of the 60 adrenal blocks available. In the remaining 51 blocks formalin pigment, neurosecretory granules, and reticulin made definitive diagnosis of bacteria very difficult. Bacteria were detected with the Gram stain in $14(23 \%)$ of 60 cases in which adrenal blocks were available. Gram-positive cocci (Figure 1b) were identified in 10 cases, Gram-negative cocci (Figure 1c) in three, and a mixed population of Gram-positive and -negative cocci and bacilli were detected in one case. By using immunohistochemistry, bacteria and bacterial antigens were detected in $40(61 \%)$ of 65 adrenal glands. 
Table 1 Antibodies used in immunohistochemical assays

\begin{tabular}{|c|c|c|c|}
\hline Antibody against & Animal source & Dilution & References \\
\hline Neisseria meningitidis $\mathrm{a}$ & Horse & $1: 500$ & 14 \\
\hline Group A streptococcus ${ }^{b}$ & Rabbit & $1: 2000$ & 15 \\
\hline Rickettsia rickettsii $^{\mathrm{c}}$ & Rabbit & $1: 500$ & 16 \\
\hline Streptococcus pneumoniae ${ }^{\mathrm{d}}$ & Rabbit & $1: 3000$ & 17 \\
\hline Staphylococcus aureus $s^{\mathrm{e}}$ & Rabbit & $1: 500$ & 18 \\
\hline Ehrlichia spp. ${ }^{\mathrm{f}}$ & Dog & $1: 100$ & 19 \\
\hline Bacillus anthracis cell wall & Mouse & $1: 200$ & 20 \\
\hline Leptospira spp..$^{\mathrm{g}}$ & Rabbit & $1: 400$ & 21 \\
\hline Clostridium spp. ${ }^{\mathrm{h}}$ & Rabbit & $1: 1000$ & 18 \\
\hline Klebsiella pneumoniae ${ }^{\mathrm{i}}$ & Rabbit & $1: 200$ & \\
\hline Legionella pneumophila & Rabbit & 1:10 000 & 22 \\
\hline Yersinia pestis & Rabbit & $1: 3000$ & 23 \\
\hline Treponema pallidum & Rabbit & $1: 200$ & 24 \\
\hline
\end{tabular}

${ }^{\mathrm{a}}$ The N. meningitidis antibody does not react with group A streptococcus (GAS), S. aureus, Listeria monocytogenes, B. anthracis, Francisella tularensis, Haemophilus influenzae, Mycoplasma pneumoniae, Pseudomonas spp., or R. rickettsii.

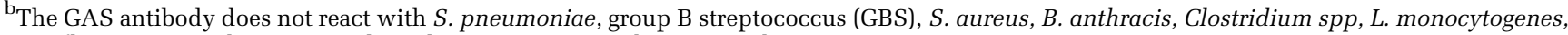
H. influenzae, Mycobacterium tuberculosis, N. meningitidis, or R. rickettsii.

${ }^{\mathrm{C}}$ Th Rickettsia spp. antibody will cross-react with various spotted fever group rickettsiae, including R. rickettsii, R. akari, R. parkeri, R. africae, and R. conorii.

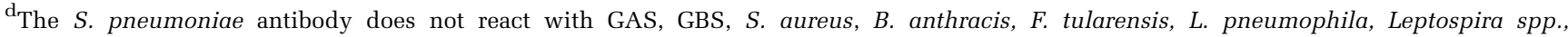
$H$. influenzae, K. pneumoniae, M. pneumoniae, M. tuberculosis, N. meningitidis, or Y. pestis.

${ }^{\mathrm{e}}$ The S. aureus antibody does not react with GAS, GBS, S. pneumoniae, B. anthracis, Clostridium spp., K. pneumoniae, Pseudomonas spp., or N. meningitidis.

${ }^{\mathrm{f}}$ The Ehrlichia spp. antibody cross-reacts with E. canis, E. chaffeensis and E. ewingii.

${ }^{\mathrm{g}}$ The Leptospira antibody is a mixture of 16 reference rabbit polyclonal anti-leptospira antisera.

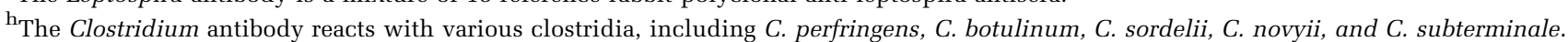

${ }^{\mathrm{i}}$ The K. pneumoniae antibody does not react with GAS, GBS, S. pneumoniae, B. anthracis, Clostridium spp., F. tularensis, H. influenzae, L. pneumophila, Leptospira spp., N. meningitidis, M. pneumoniae, or R. rickettsii.

Various amounts of bacteria and bacterial antigens were present at the site of the hemorrhage in 31 $(79 \%)$ of 39 cases with adrenal hemorrhage. Hemorrhage was considered extensive (Figure 2a) in 19 cases and focal in the other 20. Of those patients with extensive hemorrhage $15(79 \%)$ had bacteria and bacterial antigens while for those with focal hemorrhage $16(80 \%)$ had bacteria and bacterial antigens. The amount of bacteria and bacterial antigens did not correlate with the amount of hemorrhage. Adrenal hemorrhage not associated with bacterial antigens was observed in eight $(12 \%)$ cases. N. meningitidis antigens were observed in $14(36 \%)$ adrenals with hemorrhage (Figure 2b), other bacterial antigens present in adrenals with hemorrhage included group A streptococcus (three cases), S. pneumoniae (4), $R$. rickettsii (4), $S$. aureus (2), B. anthracis (2), T. pallidum (1), and L. pneumophila (1). In addition to hemorrhage, seven adrenals showed small foci of inflammation all associated with bacteria and bacterial antigens and included four cases with $R$. rickettsii and one of each $N$. meningitidis, $S$. aureus, and $S$. pneumoniae. In the cases with rickettsial infections the inflammation and bacterial antigens were primarily localized in blood vessels of the capsule and sparsely in cortical sinusoids.

Adrenal glands without hemorrhage were present in $26(40 \%)$ cases and were associated with bacteria and bacterial antigens in nine. In these nine adrenals the bacteria and bacterial antigens were associated with various pathologic lesions including small inflammatory foci in four patients (two with Ehrlichia spp. (Figure 2c), one with $R$. rickettsii (Figure 2d), and one with Leptospira spp.), foci of necrosis in one (with Leptospira spp.), and in areas of edema and congestion in the remainder four (two with group A streptococci, one with $R$. rickettsii and one with $Y$. pestis). Seventeen adrenals showed no evidence of bacteria and no hemorrhage including $N$. meningitidis (two cases), group A streptococci (2), S. pneumoniae (4), $R$. rickettsii (3), S. aureus (2), $B$. anthracis (1), Clostridium spp. (2), and $K$. pneumoniae (1). Only three of the 17 adrenal glands without hemorrhage and no evidence of bacteria or bacterial antigens showed significant pathology including a $R$. rickettsii case that had inflammatory foci and two cases with $S$. aureus septicemia that showed necrotic foci in the adrenal gland.

Of the 25 patients without bacteria in the adrenal glands, 14 had systemic disease. The cases with systemic disease but without bacteria in the adrenal glands included four cases with meningococcemia (three of them with meningitis), three cases with pneumonia due to group A streptococci, three with Rocky Mountain spotted fever, two with $S$. aureus pneumonia, one with meningitis caused by $S$. pneumoniae, and one with Ehrlichia spp. 
Table 2 Number of cases with each infection, demographic data, syndromic information and pathological findings

\begin{tabular}{|c|c|c|c|c|c|c|c|c|}
\hline $\begin{array}{l}\text { Infectious } \\
\text { agent }\end{array}$ & $\begin{array}{l}\text { No. of } \\
\text { cases }\end{array}$ & $\begin{array}{l}\text { Mean age } \\
\text { (range) }\end{array}$ & $\begin{array}{l}\text { No. of } \\
\text { males }\end{array}$ & $\begin{array}{l}\text { No. with systemic } \\
\text { disease/ no. } \\
\text { with antigens } \\
\text { in adrenals }\end{array}$ & $\begin{array}{l}\text { No. without } \\
\text { systemic disease } \\
\text { (syndrome) }\end{array}$ & $\begin{array}{c}\text { Adrenal } \\
\text { hemorrhage }\end{array}$ & $\begin{array}{c}\text { Glomerular } \\
\text { thrombi }\end{array}$ & $\begin{array}{c}\text { Lung } \\
\text { hemorrhage }\end{array}$ \\
\hline Neisseria meningitidis & 18 & $14(1-52)$ & 9 & $18 / 14$ & 0 & $16(89 \%)$ & $6 / 12$ & $1 / 12$ \\
\hline Group A streptococcus & 9 & $23(0.5-59)$ & 6 & $8 / 5$ & $\begin{array}{l}1 \text { (necrotizing } \\
\text { fasciitis) }\end{array}$ & $5(56 \%)$ & $2 / 4$ & $0 / 3$ \\
\hline Streptococcus pneumoniae & 9 & $21(1-53)$ & 7 & $5 / 4$ & 4 (pneumonia) & $5(56 \%)$ & $1 / 7$ & $0 / 7$ \\
\hline Rickettsia rickettsii & 9 & $19(1-54)$ & 6 & $9 / 6$ & 0 & $4(44 \%)$ & $1 / 7$ & $2 / 6$ \\
\hline Staphylococcus aureus & 6 & $28(5-44)$ & 3 & $4 / 2$ & 2 (pneumonia) & $4(67 \%)$ & $1 / 6$ & $2 / 6$ \\
\hline Bacillus anthracis & 3 & $69(49-94)$ & 2 & $2 / 2$ & $\begin{array}{c}1 \text { (mediastinal } \\
\text { hemorrhage) }\end{array}$ & $2(67 \%)$ & $1 / 3$ & $2 / 3$ \\
\hline Ehrlichia sp. & 3 & $42(22-66)$ & 3 & $3 / 2$ & 0 & $1(33 \%)$ & $0 / 1$ & $1 / 1$ \\
\hline Leptospira $s p$. & 2 & $28(16-40)$ & 1 & $2 / 2$ & 0 & 0 & $0 / 1$ & $1 / 1$ \\
\hline Clostridium sp. & 2 & $19(18-20)$ & 0 & 0 & $\begin{array}{l}2 \text { (necrotizing } \\
\text { endometritis) }\end{array}$ & 0 & $0 / 2$ & $1 / 2$ \\
\hline Yersinia pestis & 1 & 13 & 1 & $1 / 1$ & 0 & 0 & $1 / 1$ & $0 / 1$ \\
\hline Klebsiella pneumoniae & 1 & 6 months & 1 & 0 & 1 (pneumonia) & 0 & 0 & $0 / 1$ \\
\hline Treponema pallidium & 1 & Fetus & 0 & $1 / 1$ & 0 & 1 & 0 & 0 \\
\hline Legionella pneumophila & 1 & 44 & 1 & $1 / 1$ & 0 & 1 & $0 / 1$ & $0 / 1$ \\
\hline
\end{tabular}

\section{Discussion}

Hemorrhage was the most frequent adrenal gland pathology observed in this series of fatal bacterial infections. In our series, bacteria and bacterial antigens were seen in almost $80 \%$ of the adrenal glands with hemorrhage; however, the exact pathogenic role they play in the development of this pathology is not clear, as there are cases with adrenal gland hemorrhage without demonstrable bacteria. Adrenal hemorrhage usually occurs in the setting of a life-threatening illness, such as sepsis, in which there are low cortisol levels and consequently increased adrenocorticotropic hormone. ${ }^{1,25}$ In experimental animal models, it is necessary to have an adrenocorticotropic hormone-stimulated gland that is challenged by endotoxin or another condition such as hypovolemia to create adrenal hemorrhage. ${ }^{12,26}$ Consequently, presence of detectable bacteria in the gland is not considered essential for the occurrence of adrenal hemorrhage. In our series, the amounts of bacteria and bacterial antigens varied from patient to patient and did not correlate with the extent of hemorrhage; hence, it is possible that bacteria could have been present in areas that were not tested in those cases with hemorrhage but no bacteria in the adrenal glands. Our data suggest that the presence of bacteria in the gland may only be a consequence of the septicemia.

In a series of 22 cases with bilateral adrenal hemorrhage that included infectious and noninfectious etiologies, $59 \%$ of cases showed hemorrhages into other organs which were attributed to coagulation abnormalities including use of anticoagulants. ${ }^{1}$ It is estimated that $21 \%$ of cases with septicemia have laboratory evidence of disseminated intravascular coagulation. ${ }^{27}$ We searched for pathologic evidence of glomerular thrombi (hypercoagulable stage) and lung hemorrhage (fibrinolytic stage) in our cases. The proportion of cases in our series with thrombi or hemorrhage in other organs and adrenal hemorrhage was $36 \%$, which is higher than the frequency of disseminated intravascular coagulation in cases with septicemia. The higher frequency of lung hemorrhage in our series could be accounted for by specific pathogenetic bacterial mechanisms, that is, rickettsiae and leptospira damage vessel walls causing hemorrhages in various organs.

Although meningococci are the bacteria most commonly associated with adrenal hemorrhage, a broad collection of bacteria can cause it. In our series, $N$. meningitidis was the most frequently associated bacteria, followed by group A streptococci, $S$. pneumoniae, $R$. rickettsii, and $S$. aureus. Of the bacteria mentioned $N$. meningitides, group A streptococci, $S$. pneumoniae and $S$. aureus can circulate systemically in blood vessels and enter sinusoidal spaces of multiple organs including the adrenal gland and cause disseminated intravascular coagulation due to endotoxin production. ${ }^{2,3,5,6}$ On the other hand, rickettsiae infect endothelial cells and can cause blood vessel leakage with consequent hemorrhages in various organs. ${ }^{28}$ Independent of their pathogenic mechanism, clinical manifestations of Rocky Mountain spotted fever are similar to meningococcemia, including skin rash which together with adrenal hemorrhage is part of Waterhouse-Friderichsen syndrome. It is interesting that bacteria that have different pathogenic mechanisms can cause similar pathology: adrenal hemorrhage.

In our series, $40 \%$ of the adrenal glands did not show hemorrhage and a variety of pathologic features were observed especially if the bacteria were present in the gland. Inflammation in the area where the bacteria were located was the most frequent lesion; however, formation of small abscesses was not encountered in this series. Escherichia coli, S. aureus, 

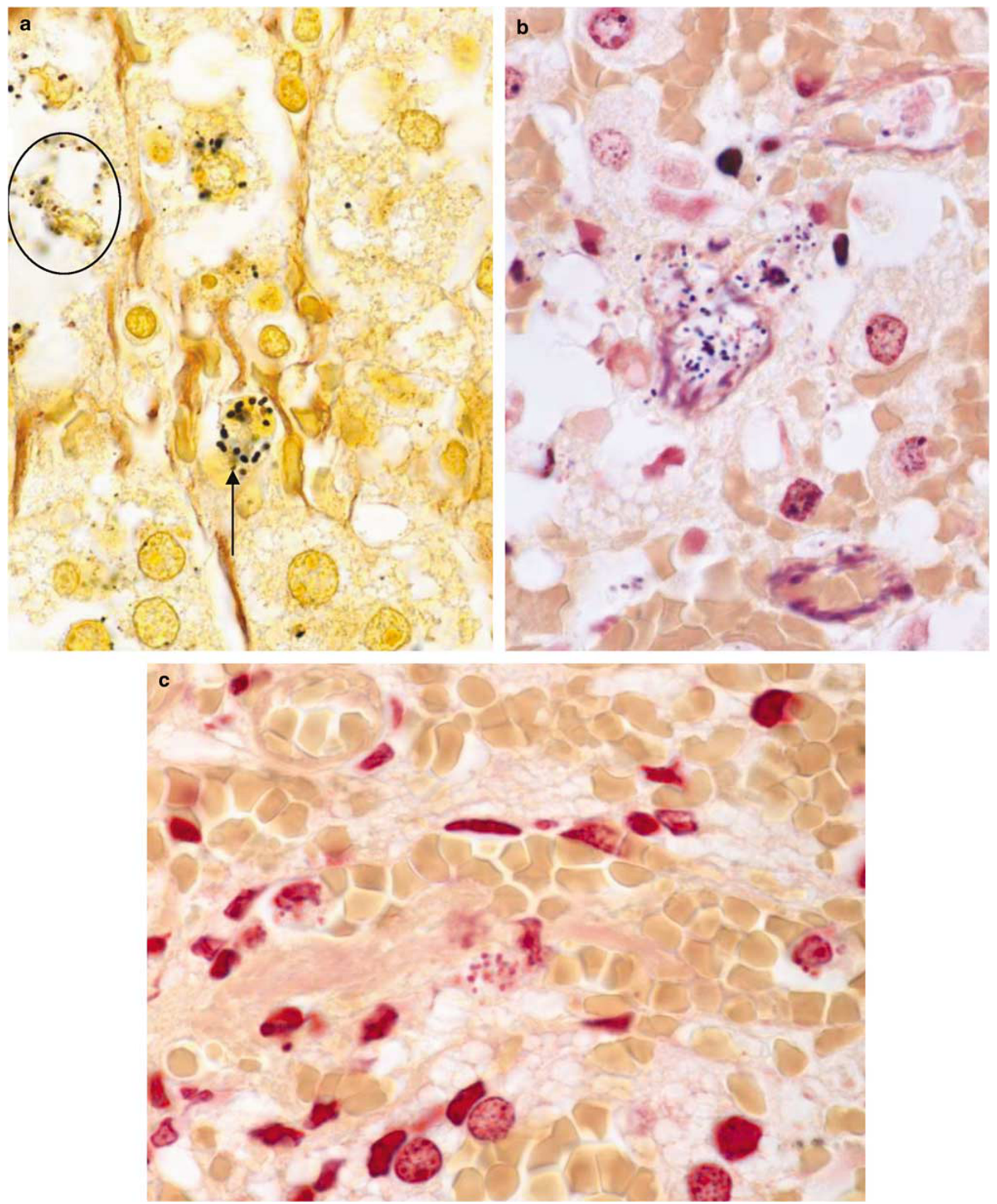

Figure 1 Special stains for bacteria including Steiner silver impregnation stain (a) showing diplococci (arrow) in a patient with invasive pneumococcal disease. Note that neurosecretory granules (circle) can make diagnosis of the bacteria difficult with silver staining techniques. Gram's stains demonstrating Gram-positive (b) and Gram-negative (c) cocci in adrenal glands of patients with invasive pneumococcal disease and meningococcemia respectively (Original magnification for $(\mathbf{a}$, $\mathbf{b}$, and $\mathbf{c} \times 250)$. 

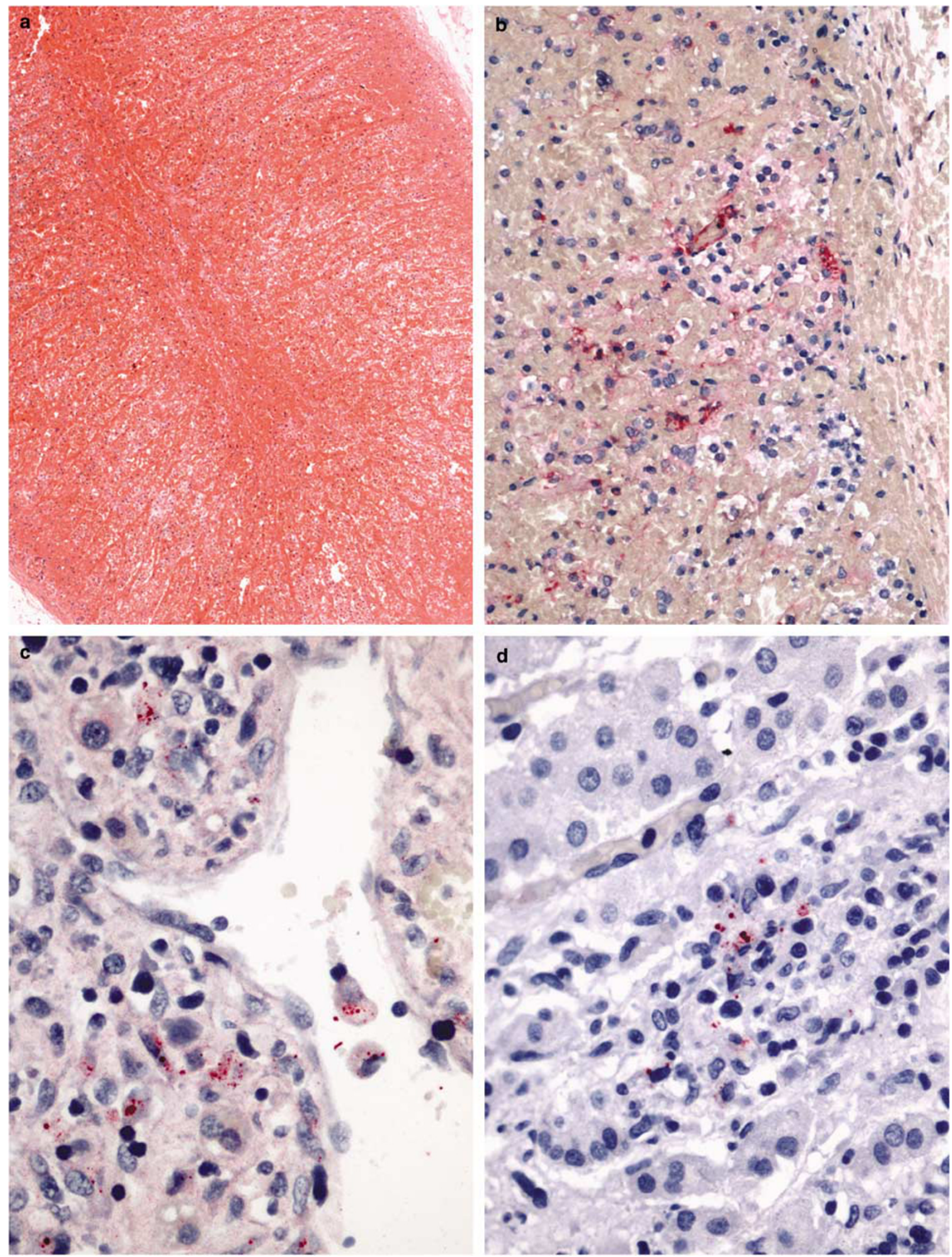

Figure 2 Extensive adrenal hemorrhage and necrosis present in a patient with meningococcemia (a). Immunohistochemical assay showing meningococci (b) in a patient with adrenal hemorrhage, Ehrlichia spp. (c) and Rickettsia rickettsii (d) in adrenals showing inflammatory foci. (a): hematoxylin and eosin stain; (b, c, and $\mathbf{d}$ ): immunohistochemical assays where primary antibodies were detected with naphthol fast red; original magnification (a) 12.5, (b) 50, (c and d) $\times 100$. 
and Candida albicans have been reported to cause adrenal gland abscesses in neonates whereas granulomas have been described in cases with Mycobacterium tuberculosis. ${ }^{29-31}$ In our series, the organisms found in the adrenal gland associated with inflammation included $R$. rickettsii, and Leptospira spp., infections that are characterized by inflammatory changes around blood vessels. ${ }^{16,22,28}$ The other organism associated with inflammatory foci in the adrenal was Ehrlichia spp. The now known Anaplasma phagocytophilum have not been previously detected in human adrenal glands of patients with granulocytic ehrlichiosis; however, they have been observed in adrenal glands of experimentally infected horses. ${ }^{32}$

The cases in our series were selected because there was an immunohistochemical diagnosis of bacterial infection. Undoubtedly, our series shows a bias because the Centers for Disease Control and Prevention receives cases of public health importance that need to be reported such as meningococcemia and those that are difficult to diagnose with routine histopathologic methods such as $R$. rickettsii, Ehrlichia spp., and Leptospira spp. In this series, $H$. influenzae infection was not diagnosed even though it has been associated with adrenal hemorrhage. ${ }^{8,10}$

Immunohistochemical assays permitted identification of intact bacteria (bacilli and cocci) and granular antigen staining. Detection of bacterial antigens using immunohistochemical assays has been observed in various bacterial infections including those caused by N. meningitidis, Clostridium spp., B. anthracis, Leptospira spp., and T. pallidum. ${ }^{14,18,20,22,24}$ In some of these infections, particularly if antibiotic therapy has been given, bacterial antigens can be more abundant than intact bacteria and in some cases antigens can be the only evidence of the causative infectious agent. These antigens probably represent break down products of the bacteria including endotoxins, which may be important contributors to adrenal hemorrhage.

This study expands on others that document adrenal hemorrhage in bacterial infections. It shows that a broad collection of bacteria can cause adrenal hemorrhage in addition to the classically associated meningococci. Immunohistochemistry allowed detection of bacterial antigens in adrenal glands with hemorrhage and may be useful to further define the pathogenic role of bacteria in this entity. Immunohistochemical assays can be of great diagnostic value, particularly for bacteria such as $R$. rickettsii, Ehrlichia spp., Leptospira spp., and Clostridium spp., which are difficult to isolate.

\section{Acknowledgement}

We thank state epidemiologists, physicians, pathologists, and medical examiners who submitted cases for consultation to the Infectious Diseases Pathology
Branch at the Centers for Disease Control and Prevention. We thank Dr Wun Ju Shieh for his advice. The work presented here was done as part of routine public health by the Centers for Disease Control and Prevention, a federal government agency. The findings and conclusions in this report are those of the authors and do not necessarily represent the views of the funding agency.

\section{References}

1 Xarli V, Steele A, Davis P, et al. Adrenal hemorrhage in the adult. Medicine 1978;57:211-221.

2 Adem P, Montgomery C, Husain A, et al. Staphylococcus aureus sepsis and the Waterhouse-Friderichsen syndrome in children. N Engl J Med 2005;353: 1245-1251.

3 Hidalgo-Carballal A, Suarez-Mier M. Sudden unexpected death in a child with varicella caused by necrotizing fasciitis and streptococcal toxic shock syndrome. Am J Forensic Med Pathol 2006;27:93-96.

4 Hori K, Yasoshima H, Yamada A, et al. Adrenal hemorrhage associated with Klebsiella oxytoca bacteremia. Intern Med 1998;37:990-994.

5 Karakousis P, Page K, Varello M, et al. WaterhouseFriderichsen syndrome after infection with group A streptococcus. Mayo Clin Proc 2001;76:1167-1170.

6 Hamilton D, Harris M, Foweraker J, et al. WaterhouseFriderichsen syndrome as a result of non-meningococcal infection. J Clin Pathol 2004;57:208-209.

7 Bohm N. Adrenal, cutaneous and myocardial lesions in fulminating endotoxinemia (Waterhouse-Friderichsen syndrome). Pathol Res Pract 1982;174:92-105.

8 Jacobs R, Hsi S, Benjamin CWD, et al. Apparent meningococcemia: clinical features of disease due to Haemophilus influenzae and Neisseria meningitidis. Pediatrics 1983;72:469-472.

9 Swierczewski J, Mason E, Cabrera P, et al. Fulminating meningitis with Waterhouse-Friderichsen syndrome due to Neisseria gonorrhoeae. Am J Clin Pathol 1970;54:202-204.

10 Dyke M, Martin R, Berry P. Septicaemia and adrenal haemorrhage in congenital asplenia. Arch Dis Child 1991;66:636-637.

11 Levi M, Keller T, Grop Ev, et al. Infection and inflammation and the coagulation system. Cardiovasc Res 2003;60:26-39.

12 Aoyama H, Kikuchi F, Mori W. Acute, massive, haemorrhagic adrenal necrosis experimentally produced by the Shwartzman mechanism in rabbits. Virchows Arch A Pathol Anat Histopathol 1987;412: 11-16.

13 Danielsson D, Nathorst-Windahl G, Salden T. Use of immunofluorescence for identification of Haemophilus influenzae and Neisseria meningitidis in postmortem human tissue. Ann N Y Acad Sci 1971;177:23-31.

14 Guarner J, Greer P, Whitney A, et al. Pathogenesis and diagnosis of human meningococcal disease using immunohistochemical and PCR assays. Am J Clin Pathol 2004;122:754-764.

15 Guarner J, Sumner J, Paddock C, et al. Diagnosis of invasive group A streptococcal infections by using immunohistochemical and molecular assays. Am J Clin Pathol 2006;126:148-155. 
16 Paddock C, Greer P, Ferebee T, et al. Hidden mortality attributable to Rocky Mountain spotted fever: immunohistochemical detection of fatal, serologically unconfirmed disease. J Infect Dis 1999;179:1469-1476.

17 Guarner J, Packard M, Nolte K, et al. Utility of immunohistochemical diagnosis of Streptococcus pneumoniae in formalin-fixed, paraffin-embedded specimens compared to culture and Gram's stains techniques. Am J Clin Pathol 2006;127:612-618.

18 Guarner J, Bartlett J, Reagan S, et al. Immunohistochemical evidence of Clostridium sp., Staphylococcus aureus, and group A streptococcus in severe soft tissue infections related to injection drug use. Hum Pathol 2006;37:1482-1488.

19 Dawson J, Paddock C, Warner C, et al. Tissue diagnosis of Ehrlichia chaffeensis in patients with fatal ehrlichiosis by use of immunohistochemistry, in situ hybridization, and polymerase chain reaction. Am J Trop Med Hyg 2001;65:603-609.

20 Guarner J, Jernigan J, Shieh W, et al. Pathology and pathogenesis of bioterrorism-related inhalational anthrax. Am J Pathol 2003;163:701-709.

21 Zaki S, Shieh W, Group EW. Leptospirosis associated with outbreak of acute febrile illness and pulmonary haemorrhage, Nicaragua, 1995. Lancet 1996;347: 535-536.

22 Fiore A, Nuorti J, Levine O, et al. Epidemic Legionnaires' disease two decades later: old sources, new diagnostic methods. Clin Infect Dis 1998;26: 426-433.

23 Guarner J, Shieh W, Greer P, et al. Immunohistochemical detection of Yersinia pestis in formalin-fixed, paraffin-embedded tissue. Am J Clin Pathol 2002;117: 205-209.
24 Guarner J, Southwick K, Greer P, et al. Testing umbilical cords for funisitis due to Treponema pallidum infection, Bolivia. Emerg Infect Dis 2000;6:487-492.

25 Brinker $\mathrm{Md}$, Joosten $\mathrm{K}$, Liem $\mathrm{O}$, et al. Adrenal insufficiency in meningococcal sepsis: bioavailable cortisol levels and impact of interleukin-6 levels and intubation with etomidate on adrenal function and mortality. J Clin Endocrinol Metab 2005;90: 5110-5117.

26 Rushing G, Britt R, Britt L. Effects of hemorrhagic shock on adrenal response in a rat model. Ann Surg 2006;243:652-656.

27 Cauchie P, Cauchie C, Zouaoui-Boudjeltia K, et al. Diagnosis and prognosis of overt disseminated intravascular coagulation in a general hospital -meaning of the ISTH score system, fibrin monomers, and lipoprotein-C-reactive protein complex formation. Am J Hematol 2006;81:414-419.

28 Walker D, Dumler J. Rickettsial infections, Vol. 1, Ist edn. Appleton and Lange: Stamford, CT, 1997, pp 789-799.

29 Steffens J, Zaubitzer T, Kirsch W, et al. Neonatal adrenal abscesses. Eur Urol 1997;31:347-349.

30 Kwon H, Kim S, Yoo S, et al. Adrenal tuberculosis in Cushing's disease with bilateral macronodular adrenocortical hyperplasia. Endocr J 2006;53: 219-223.

31 Ratnavel N, Farrer K, Sharland M, et al. Neonatal adrenal abscess revisited: the importance of raised inflammatory markers. Ann Trop Paediatr 2005;25: 63-66.

32 Chang Y, Novosel V, Dubovi E, et al. Experimental infection of the human granulocytic ehrlichiosis agent in horses. Vet Parasitol 1998;78:137-145. 